\title{
Dyspnea Management
}

National Cancer Institute

\section{Source}

National Cancer Institute. Dyspnea Management. NCI Thesaurus. Code C15738.

Treatment to manage or alleviate difficult or painful breathing or shortness of breath. 